\title{
El impacto del nivel educativo alcanzado en el índice de calidad del empleo en el Perú, 2016
}

\section{Oscar Niquen Lasteros}

Dirección de Investigación Socio Económico Laboral

del Ministerio de Trabajo y Promoción del Empleo oniquen@trabajo.gob.pe

Recibido: 04/09/2018

Aprobado: 25/07/2019 


\section{El impacto del nivel educativo alcanzado en el índice de calidad del empleo en el Perú, 2016}

\section{Resumen}

El presente estudio de investigación tiene como objetivo medir el impacto de un mayor nivel educativo alcanzado en el índice de calidad de empleo teniendo en cuenta las brechas de género, área y categoría ocupacional. El estudio es de tipo cuantitativo con datos de corte transversal obtenidos de la Encuesta Nacional de Hogares elaborada por el Instituto Nacional de Estadística e Informática (INEI). La metodología empleada consiste en un modelo probit, ordenado para cuatro categorías del índice de empleo. Entre los principales resultados, se encontró que en el Perú la categoría con mayor probabilidad (42\%) correspondió a mala calidad de empleo y que un mayor nivel educativo alcanzado redujo en $1,9 \%$ la probabilidad de pertenecer a dicha categoría. También, se identificó que los trabajadores con educación básica tienen mayores probabilidades de tener un empleo de mala y muy mala calidad. En comparación con un trabajador con educación superior no universitaria, tienen aproximadamente tres veces menos probabilidad de pertenecer a un empleo de muy buena calidad. Otro resultado importante es que mayores niveles educativos no presentaron menores brechas en género y categoría ocupacional. Si bien la probabilidad de tener buena calidad de empleo de hombres y mujeres aumentó en cada nivel educativo, del mismo modo, incrementó la diferencia entre probabilidades. Lo mismo ocurrió entre asalariados e independientes, marco en el que los asalariados tuvieron mayor beneficio al cualificarse en comparación con los independientes.

Palabras clave: Educación, análisis socioeconómico, economía del trabajo

\section{The impact of the educational level achieved in the index of quality of employment in Peru, 2016}

\section{Abstract}

The objective of this research study is to measure the impact of higher level of education in the employment quality index, considering gender, area, and occupational category gaps. This quantitative study analyzes cross-sectional data obtained from the National Household Survey prepared by the INEI. The methodology consists in an ordered probit model for four categories of the employment index. Among the main results, it was found that, in Peru, the category of poor quality of employment is the one with the highest probability (42.0\%), while a higher educational level reduces the probability of belonging to this category. Also, the research shows that workers with basic education are more likely to have a bad and very poor-quality job. Compared to a worker with non-university higher education, they are approximately three times less likely to belong to a very good quality job. Another important outcome is that higher educational levels do not show minor gaps in gender and occupational category. Although the probability of women and men having good quality employments increases in each educational level, the difference between probabilities also did. The same occurred between employees and independents; in this case, salaried employees have a greater benefit in qualifying compared to independent workers.

Keywords: Education, socio-economic analysis, labor economics 


\section{Introducción}

En los últimos años, las instituciones encargadas de la política pública laboral ya no se han enfocado exclusivamente en la reducción del desempleo, sino que han tratado de armonizar la reducción del paro con la creación de nuevos puestos de trabajo con un mínimo de calidad requerida, lo que ha derivado en un tema complejo de abordar (Gonzales y Mora, 2011). Esta búsqueda de mejores puestos de trabajos se ve justificada por la evidencia empírica. Por ejemplo, en el caso peruano, la tasa de desempleo para el 2016 fue de apenas $4,2 \%$. Sin embargo, para el mismo año, el $72 \%$ de la población ocupada se encontró laborando informalmente, lo que evidencia la gran brecha existente en el mercado laboral.

Esta brecha observada trajo consigo metodologías destinadas a medir las condiciones laborales. Entre ellas, destaca el índice de calidad del empleo (ICE), que, mediante una escala del uno al cien, toma referencia sobre la calidad laboral en la que trabaja un asalariado o independiente. Para el caso de Perú, en el 2016, la puntuación alcanzada fue de 40,9, lo que significa una mala calidad del empleo (teniendo en cuenta indicadores económicos, contractuales y sociales). Cabe destacar que, para el 2016, solo la región de Moquegua tuvo un puntaje mayor a 50,0 (50,5), situándose como la única con buena calidad de empleo.

Latinoamérica tampoco ha sido ajena a esta brecha de calidad laboral. El estudio de Weller y Rothlisbeger (2011) encuentra un déficit en la mayoría de los países en materia de calidad de empleo, considerando aspectos como los ingresos, la afiliación a salud, la garantía de derecho laboral, los beneficios, etc. Así pues, esta arraigada problemática internacional ha ocasionado que los investigadores empiecen a darle la debida importancia al análisis formal de los determinantes del empleo de calidad.

En este contexto, la variable que más ha destacado en el estudio empírico ha sido el nivel educativo, gracias a que se presenta como una variable que ha tenido resultados positivos y significativos en las estimaciones. Por dar algunos ejemplos, estudios como el de Mora y Ulloa (2011) encuentran que un año adicional de educación mejora el índice de calidad en 1,4 veces. De la misma manera, Gaviria y Quingua (2015) hallan que un aumento de $1 \%$ en los años de educación ocasiona que la calidad de empleo mejore, en promedio, en $0,67 \%$. La evidencia empírica, en ese sentido, daría pie a la importancia de medir los efectos del nivel educativo en el índice de calidad del empleo del Perú. En el país, hasta el año 2016, solo el 11,6\% de la PEA (población económicamente activa) ocupada asalariada e independiente tuvo educación superior universitaria, el 10,8\% accedió a educación superior no universitaria y el grueso de trabajadores tuvo nivel alcanzado hasta educación secundaria (40,1\%). Es importante mencionar que la teoría detrás de la relación entre calidad de trabajo y educación es la teoría del capital humano, planteada por Becker (1964). Sobre esta base, el presente artículo de investigación no solo busca cuantificar el índice de calidad del empleo (ICE), sino también pretende medir el impacto de sus 
principales determinantes de una manera formal, teniendo como foco principal el nivel educativo alcanzado de los trabajadores.

\section{Revisión de la literatura}

En un primer momento, los atributos que se consideraron para medir la calidad del trabajo fueron el estatus de trabajo, la seguridad en el empleo, y la afiliación a seguro y pensiones (Rosenthal, 1989). Luego, Verdera (1995) incluyó en los indicadores tradicionales la duración de la jornada de trabajo, la sindicalización y la estabilidad laboral. Posteriormente, Valenzuela (2000) tomó en consideración el balance trabajo/familia y las características de la jornada de trabajo. Más tarde, entre los trabajos que mejor lograron aproximar un índice de calidad laboral tomando en cuenta el beneficio social y económico condicionado a las normas institucionales, se encuentra el estudio de Farné (2003). Este autor resumió el índice en cuatro variables básicas: la afiliación a seguro de salud y sistema de pensiones, el ingreso laboral, el tipo de contrato y las horas trabajadas. Dependiendo de si el trabajador era asalariado o independiente, Farné le atribuyó ponderaciones específicas a cada una de estas variables.

En el Perú, solo existen reportes de la evolución del índice de calidad del empleo (ICE) elaborados por el Ministerio de Trabajo y Promoción del Empleo (MTPE) siguiendo la propuesta de Farné (2003); no hay investigaciones que midan el impacto del nivel educativo de las personas ni de ninguno de sus determinantes. Tampoco, hay estudios sobre las brechas existentes de las características socioeconómicas en el acceso al empleo de calidad, lo que justificaría la realización del presente artículo de investigación.

Enfocándose en Latinoamérica, el estudio realizado por Weller y Roethlisberger (2011) concluye que existe una elevada heterogeneidad estructural, una desigualdad en la distribución de los activos y una mala calidad en el nivel educativo, lo que genera una baja productividad laboral. Los autores, también, sugieren que los efectos agregados y la institucionalidad laboral no son los únicos factores determinantes, sino que existen brechas segmentadas como el área geográfica y el género.

En el caso de Colombia, existen diversos estudios sobre la relación entre el nivel educativo y el mercado laboral. Entre ellos, destaca el estudio realizado por Mora y Ulloa (2011), que midió la relación entre el índice de calidad del empleo y el nivel educativo mediante un modelo logit multinomial. A partir de ello, se identificó que un año adicional de educación aumentó en 1,4 veces la probabilidad de tener un empleo de calidad alta sobre un empleo de calidad baja. De igual manera, se encontró que aún persisten las brechas de género y las brechas entre ciudades para el acceso al empleo de calidad.

Otro estudio colombiano importante sobre el índice de calidad del empleo es el realizado por Gaviria y Quingua (2015), quienes, por medio de elasticidades, determinaron que un aumento de $1 \%$ en los años de escolaridad aumentaría la calidad del empleo en $0,67 \%$ (efecto inelástico). También, identificaron 
que las mujeres tienen, en promedio, un menor índice que los hombres y que la edad tiene un efecto positivo en la calidad del empleo, aunque con una elasticidad baja $(0,47 \%)$.

De igual forma, la investigación realizada por Riomaña (2014) sobre la incidencia de la educación en el empleo y el desempleo en Colombia concluye que un mayor nivel educativo reduce la probabilidad de ser un desempleado de corta duración. De manera contraria, ser jefe de hogar y ser hombre aumenta las probabilidades de ser un desempleado de corta duración.

En España, los estudios realizados por Pastor, Raymond, Roig y Serrano (2008) hallaron que la probabilidad de empleo de un hombre de 60 años aumenta más de $20 \%$ si es licenciado. Además, identificaron que la diferencia entre sexos en la posibilidad de tener empleo disminuye cuando el nivel educativo es mayor; y, por último, que una alta cualificación implica un mayor salario y una mayor probabilidad de ser empleado, por lo que se traduce en un mayor rendimiento.

Por su parte, según la investigación de Cueto y Pruneda (2014), también enfocada en España, el 50\% de desempleados carece de estudios especializados. Sobre esta base, los autores concluyen que las expectativas de tener un empleo son considerablemente mejores para quienes poseen educación especializada que para los que no la tienen. Asimismo, señalan que, a pesar de las coyunturas económicas adversas, los trabajadores con estudios universitarios son quienes tienen mejor posición dentro del mercado de trabajo. Estos resultados dejan entrever el riesgo laboral que poseen los trabajadores con baja cualificación.

\section{Teoría del capital humano}

La principal teoría que vincula el nivel educativo y el trabajo es la del capital humano planteado por Schultz (1961) y Becker (1964). En ella, se expone que la educación es un proceso de inversión que realizan las personas de manera racional en el presente con la finalidad de obtener en el futuro empleos de calidad y altos cargos con grandes ingresos laborales (Riomaña, 2014). Es importante señalar que, según esta teoría, la decisión de invertir en educación está sujeta a un análisis de costo de oportunidad. Es decir, si el costo de inversión es mayor al costo de oportunidad, el individuo tendrá incentivos para seguir educándose (Alva, 2016). Asimismo, Becker (1984) adujo que la relación directa entre dichas variables se debía a que la educación y el conocimiento aumentaban la eficiencia de los trabajadores, haciendo que puedan aspirar a empleos de mejor calidad. Finalmente, el autor reforzó esta teoría encontrando una fuerte relación entre la educación y el progreso económico.

\section{Metodología}

La investigación tiene un tipo de enfoque cuantitativo. El diseño utilizado es no experimental y los datos son de corte transversal. Para el cálculo del índice 
de calidad del empleo, se usó la metodología propuesta por Farné (2003), empleada en investigaciones como las de Mora y Ulloa (2011), y Gaviria y Quingua (2015). Dicha metodología, además, es utilizada en los reportes de empleo del Ministerio de Trabajo y Promoción del Empleo (MTPE) del Perú (2016).

Las variables para calcular el índice son el ingreso laboral promedio mensual, el tipo de contrato (medida de estabilidad laboral), seguridad social (medida por afiliación a seguro de salud y sistema de pensiones) y horas de trabajo semanales (proxy de balance entre familia/trabajo). Cabe anotar que la inclusión del ingreso laboral promedio mensual es justificada debido a la información que brinda sobre la capacidad de consumo y el mayor acceso a servicios que cubran necesidades básicas.

Siguiendo con la metodología propuesta por los autores mencionados anteriormente, se determinó la siguiente asignación de puntajes para las variables:

Tabla 1. Asignación de puntajes de las variables del índice de calidad del empleo

\begin{tabular}{|c|c|}
\hline Variables & Asignación de puntajes \\
\hline Ingreso laboral promedio mensual & $\begin{array}{l}\text { - } 0 \text { puntos si el salario es menor a } 1,5 \text { veces la } \\
\text { Remuneración Mínima Vital. } \\
\text { - } 50 \text { puntos si el salario oscila entre } 1,5 \text { y } 3 \text { veces } \\
\text { la RMV. } \\
\text { - } 100 \text { puntos si el ingreso laboral es mayor a } 3 \\
\text { veces la RMV. }\end{array}$ \\
\hline Tipo de contrato & $\begin{array}{l}\text { - } 0 \text { puntos para los trabajadores sin contrato } \\
\text { laboral. } \\
\text { - } 50 \text { puntos si el contrato es temporal. } \\
\text { - } 100 \text { puntos si el ingreso laboral es mayor a } 3 \\
\text { veces la RMV. }\end{array}$ \\
\hline Seguridad social & $\begin{array}{l}\text { - } 0 \text { puntos si el empleado no cuenta con afiliación } \\
\text { a seguro de pensiones ni a seguro de salud. } \\
\text { - } 50 \text { puntos si el trabajador posee una de las dos } \\
\text { afiliaciones. } \\
\text { - } 100 \text { puntos si posee ambas afiliaciones. }\end{array}$ \\
\hline Horas de trabajo semanales & $\begin{array}{l}\text { - } 0 \text { puntos si el trabajador lanora más de } 48 \text { horas } \\
\text { a la semana. } \\
\text { - } 100 \text { puntos si trabaja igual o menos de } 48 \text { horas } \\
\text { a la semana. }\end{array}$ \\
\hline
\end{tabular}

Fuente: Farné, 2003. Elaboración propia.

De igual manera, al igual que en los estudios de Farné (2003), Bustamante y Arroyo (2008), Posso (2010) y el MTPE (2016), se consideró si se trataba de trabajadores asalariados o trabajadores independientes. Las ponderaciones 10 I fueron las siguientes: 
Tabla 2. Ponderación de variables según tipo de ocupación

\begin{tabular}{ccc}
\hline Variables & Asalariados & Independientes \\
\hline Ingreso laboral promedio mensual & $40 \%$ & $50 \%$ \\
Tipo de contrato & $25 \%$ & - \\
Seguridad social & $25 \%$ & $35 \%$ \\
Horas de trabajo semanales & $10 \%$ & $15 \%$ \\
\hline
\end{tabular}

Fuente: Farné, 2003. Elaboración propia.

Por último, para la interpretación del índice, se siguió lo recomendado por Farné (2003) y el MTPE del Perú (2016), como se muestra en la siguiente tabla.

Tabla 3. Puntaje e interpretación del ICE

\begin{tabular}{cc}
\hline Puntaje & Interpretación \\
\hline Menor a 25 puntos & Muy mala calidad \\
Entre 25 y 50 puntos & Mala calidad \\
Entre 50 y 75 puntos & Buena calidad \\
Más de 75 puntos & Muy buena calidad \\
\hline
\end{tabular}

Fuente: MTPE, 2016. Elaboración propia.

El modelo econométrico a utilizar fue un probit ordenado, debido a que la variable endógena expresa implícitamente un orden de utilidad —es decir, es de carácter ordinal (Rodríguez y Cáceres, 2007) - y los residuos siguen una distribución normal estandarizada.

De esta manera, la ecuación inicial es la siguiente:

$$
y^{*}=\beta^{\prime} x+\varepsilon \ldots(1)
$$

En dicha ecuación, la variable $y^{*}$ es no observable. Además, el modelo probit ordenado supone una variable endógena discreta mediante la especificación detallada a continuación:

$$
\begin{gathered}
y_{i}=0 \text { si } y_{i}{ }^{*}<\gamma_{1} \\
y_{i}=1 \text { si } \gamma_{1} \leq y_{i}^{*}<\gamma_{2} \\
y_{i}=2 \text { si }<\gamma_{2}{ }^{*}<\gamma_{i}
\end{gathered}
$$

En esta fórmula, $\beta$ y $\gamma$ son los parámetros del modelo. Los $\gamma$ son los que representan los umbrales que determinan el valor de para el valor alcanzado por $y_{i}^{*}$. Finalmente, la probabilidad de cada alternativa es la siguiente: 


$$
\begin{gathered}
\operatorname{Pr}\left[y_{i}=0\right]=\operatorname{Pr}\left[y_{i}^{*}<\gamma_{1}\right]=\operatorname{Pr}\left[\mathrm{X}_{\mathrm{i}} \beta+u_{i}<\gamma_{1}\right]=\Phi\left(\gamma_{1}-X_{i} \beta\right) \\
\operatorname{Pr}\left[y_{i}=1\right]=\operatorname{Pr}\left[\gamma_{i} \leq y_{i}^{*}<\gamma_{2}\right]=\operatorname{Pr}\left[\gamma_{i}<X_{i} \beta+u_{i}<\gamma_{2}\right]=\Phi\left(\gamma_{2}-X_{i} \beta\right)-\Phi\left(\gamma_{1}-X_{i} \beta\right) \\
\operatorname{Pr}\left[y_{i}=2\right]=\operatorname{Pr}\left[y_{i}^{*} \geq \gamma_{2}\right]=\operatorname{Pr}\left[X_{i} \beta+u_{i}<\gamma_{2}\right]=\Phi\left(X_{i} \beta-\gamma_{2}\right)
\end{gathered}
$$

Dichas probabilidades se integran en una única expresión para ser estimadas por el método de máxima verosimilitud (Rodríguez y Cáceres, 2007).

Para esta investigación, la variable dependiente tomó cuatro categorías: 1 si el puntaje del ICE fue menor a 25; el valor de 2 si estuvo entre 25 y 50 ; 3 si se encontró entre 50 y 75 ; y el valor de 4 si el puntaje fue mayor a 75 (siguiendo el puntaje e interpretación mostrado en la Tabla 3 ).

Entre las variables explicativas, se consideró el nivel educativo alcanzado ${ }^{1}$ (sin nivel, primaria incompleta, primaria completa, secundaria incompleta, secundaria completa, no universitaria incompleta, no universitaria completa, universitaria incompleta y universitaria completa), así como el sexo, la edad, el área geográfica (urbano o rural), el tipo de categoría ocupacional (asalariado o independiente) y la rama de actividad económica en la que se desempeñó el trabajador (extractiva, industria, comercio, construcción y servicios). Estas variables fueron tomadas en cuenta de acuerdo con lo sugerido en los estudios empíricos presentados en el capítulo de la revisión de la literatura.

Además, se consideró el potencial problema de endogeneidad que podría presentar la variable educación con el ICE, debido a que dicho índice contiene la variable salarios (Mora y Ulloa, 2011). El mencionado problema de endogeneidad entre la variable educación e ingresos es sustentado por la teoría del capital humano (Becker, 1964) y la teoría del escudriñamiento (Spence, 2002). Bajo esta problemática, se estimó un modelo auxiliar que permitiera contrastar la existencia de endogeneidad entre las variables en cuestión para luego, de ser necesario, corregir el sesgo de la estimación. De este modo, se estimó mediante un modelo probit ordinal ${ }^{2}$ la siguiente ecuación:

$$
\text { Educación }_{i}=Z_{i}^{\prime} \gamma+v_{i} \ldots
$$

La variable educación será considerada exógena si la correlación entre $v_{i}$ y $\varepsilon$ es cero. En caso contrario, se estimará un modelo multiecuacional de dos etapas, en que la variable educación sería instrumental (ver ecuación 2).

1. Una de las razones por las que se eligió este indicador y no el de años de educación fue porque este último no toma en consideración el carácter acumulativo de la educación (Hanushek, 2008). Por otro lado, una de las desventajas de usar el nivel educativo alcanzado es que esta variable no contempla la calidad de enseñanza ni otros aspectos como la experiencia de aprendizaje (Rivera, 2017).

2. Al igual que con el modelo principal, el criterio para elegir el modelo respondió a que la variable endógena (educación) expresa implícitamente un orden de utilidad por cada nivel alcanzado. 
Las variables usadas para explicar la educación son el estado civil (variable dummy que toma el valor de uno si es casado o conviviente, y cero en otro caso), un proxy de acceso a la educación (si vive en Lima Metropolitana, costa norte, costa centro, costa sur, sierra norte, sierra centro, sierra sur o selva), la cantidad de miembros del hogar menores de 14 años, y una variable dummy que especifica si el trabajador es jefe de hogar o no. Estas variables fueron consideradas por lo sugerido en los siguientes estudios: Endogeneidad y rendimientos de la educación de Barceinas (2002), "Las ecuaciones de Mincer y las tasas de rendimiento de la educación en Galicia" de Freire y Tejeiro (2010), y "Endogeneidad de los rendimientos económicos en México" de Villarreal (2018).

\section{Análisis de datos y resultados}

La fuente para la obtención de datos fue la Encuesta Nacional de Hogares sobre Condiciones de Vida y Pobreza (Enaho) elaborado por el Instituto Nacional de Estadística e Informática (INEI) para el año 2016. La población objetivo está conformada por la población ocupada asalariada e independiente del Perú. La muestra está conformada por 57,117 trabajadores.

Se puede observar en el Gráfico 1 que, en el Perú, los trabajadores asalariados e independientes, en promedio, tienen una mala calidad del empleo (40,9 puntos) y que 23 departamentos comparten el mismo rango de índice. Los departamentos con los menores índices de calidad son Puno (32.1), seguido de Cajamarca (32.7), Apurímac (33.0) y Amazonas (33.2). Los departamentos que están por encima del promedio nacional son Arequipa (42.2), Ica (43.2), Lima (48.5) y Moquegua (50.5). Este último fue el único que logró presentar un índice de buena calidad del empleo.

Gráfico 1. Perú: Índice de calidad del empleo por departamentos, 2016

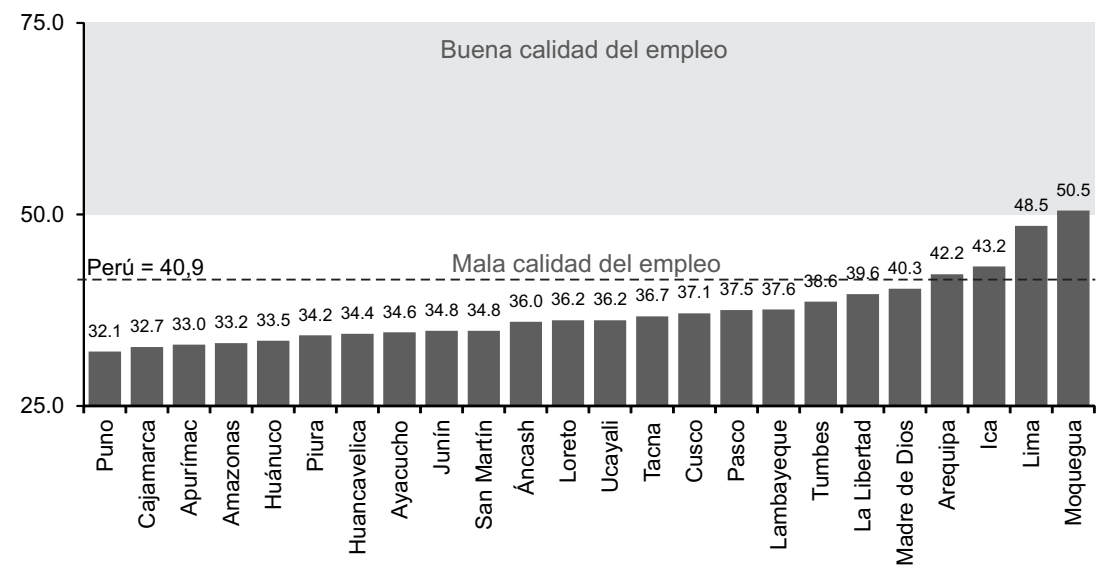

Nota: Lima incluye a la Provincia Constitucional del Callao. 


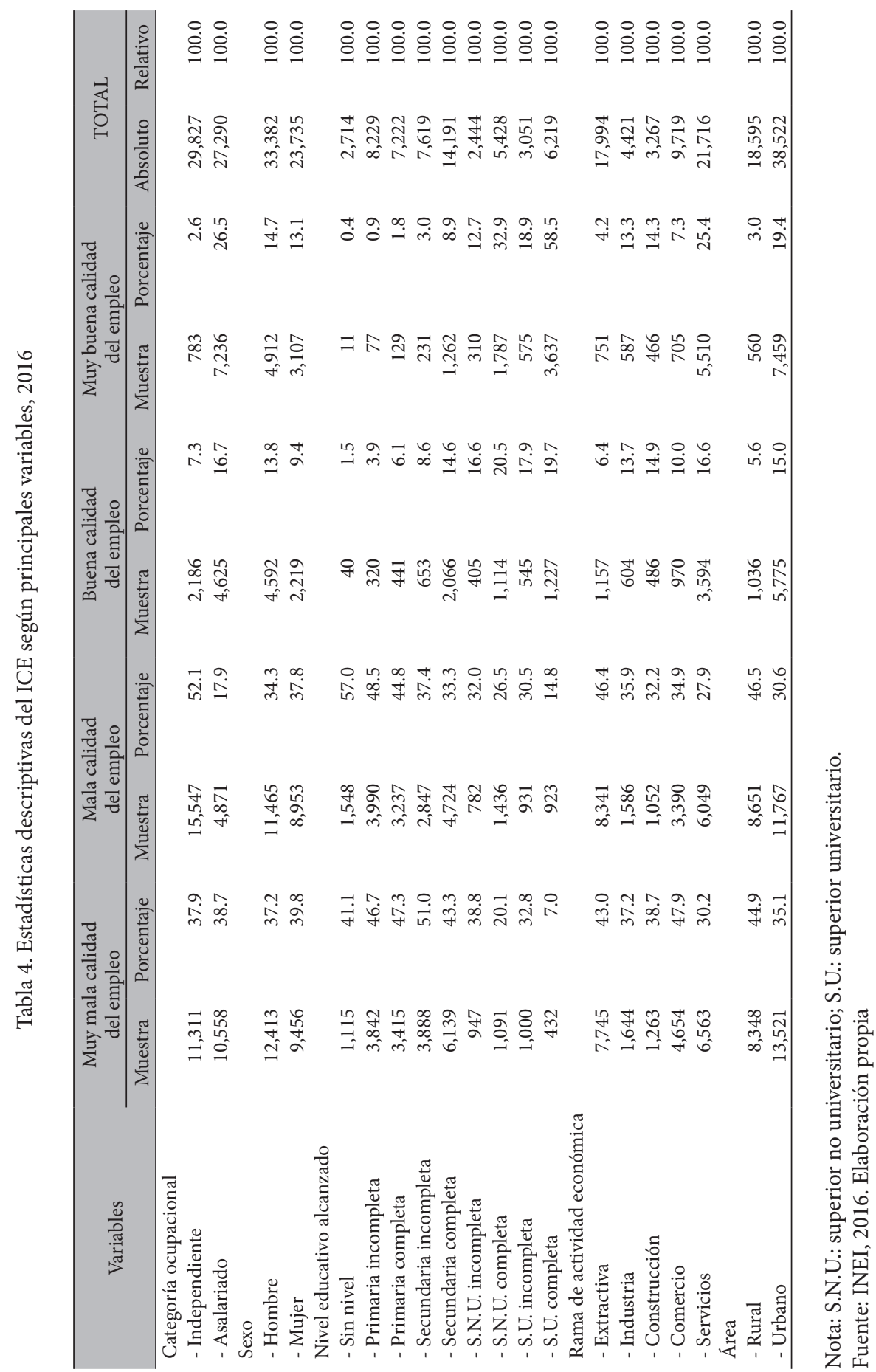


En la comparación del ICE con el nivel educativo culminado, se aprecia en el Gráfico 2 una relación positiva, un mayor nivel educativo culminado, en promedio, presentó una mayor calidad del empleo, donde la educación superior universitaria y no universitaria lograron tener una buena calidad de empleo. Cabe resaltar que entre las personas sin nivel educativo y las personas con nivel primario hubo una brecha de calidad muy baja (2.6 puntos).

Gráfico 2. Perú: Índice de calidad del empleo según nivel educativo culminado, 2016

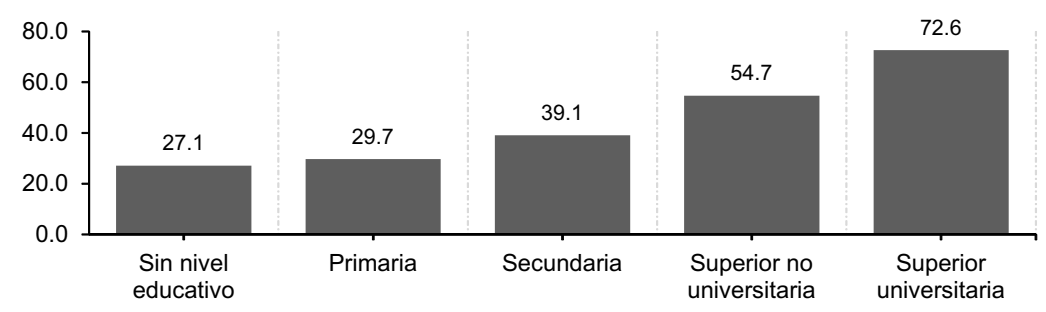

Fuente: INEI, 2016. Elaboración propia.

Con respecto al ingreso laboral promedio mensual, se observa en el Gráfico 3 que aún hay presencia de fuertes brechas entre regiones. El ingreso laboral mensual del Perú fue de 1371 soles. Las regiones con menor ingreso fueron Huancavelica (S/ 734), Cajamarca (S/ 808), Puno (S/ 857) y Ayacucho (S/ 880), mientras que las regiones con mayores ingresos fueron Lima (S/ 1870), Moquegua (S/ 1818) y Madre de Dios (S/ 1632).

Gráfico 3. Perú: Ingreso laboral promedio mensual de asalariados e independientes, 2016

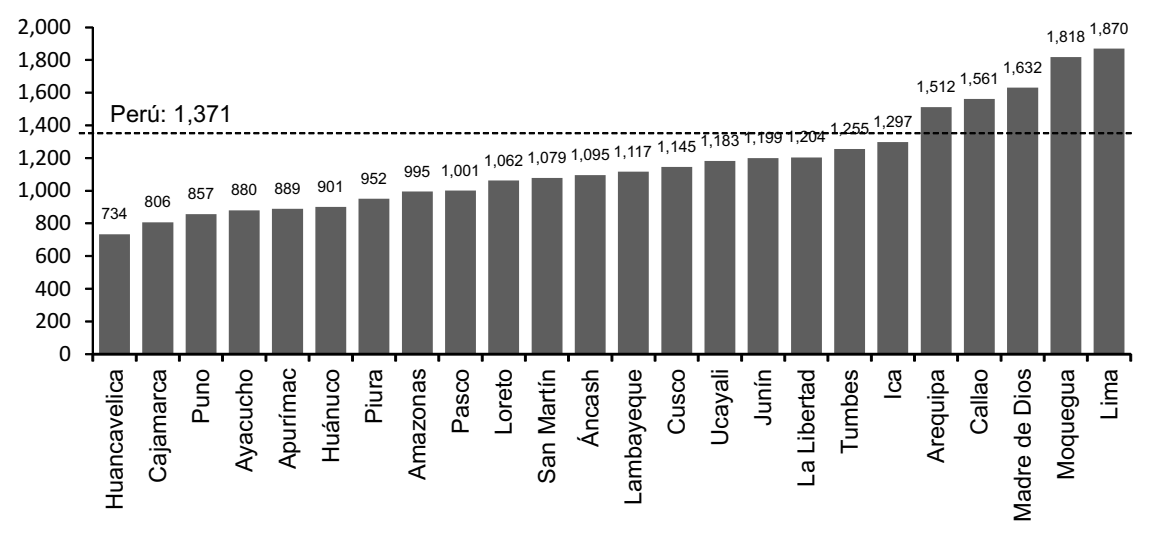

Fuente: INEI, 2016. Elaboración propia. 
El Gráfico 4 vuelve a mostrar una incidencia positiva de la educación, esta vez con respecto al ingreso laboral mensual. Un mayor nivel educativo ha estado relacionado con mayores ingresos laborales, marco en el que la educación superior universitaria presenta los niveles más altos, con una gran brecha incluso en relación con la educación superior no universitaria.

Tomando en cuenta el tipo de contrato, el Gráfico 5 muestra que el 80,3\% de la PEA ocupada asalariada e independiente con educación superior universitaria contó con contrato indefinido o a plazo fijo, mientras que para los empleados con secundaria completa la cifra se redujo a casi la mitad (44.2\%). Asimismo, se observa que el $82.7 \%$ de las personas sin nivel educativo laboraron sin contrato, cifra que disminuyó considerablemente para cada nivel educativo mayor alcanzado, hasta representar solo el 7\% de los trabajadores con educación superior universitaria.

Gráfico 4. Perú: Ingreso laboral promedio mensual de asalariados e independientes, según nivel educativo culminado, 2016

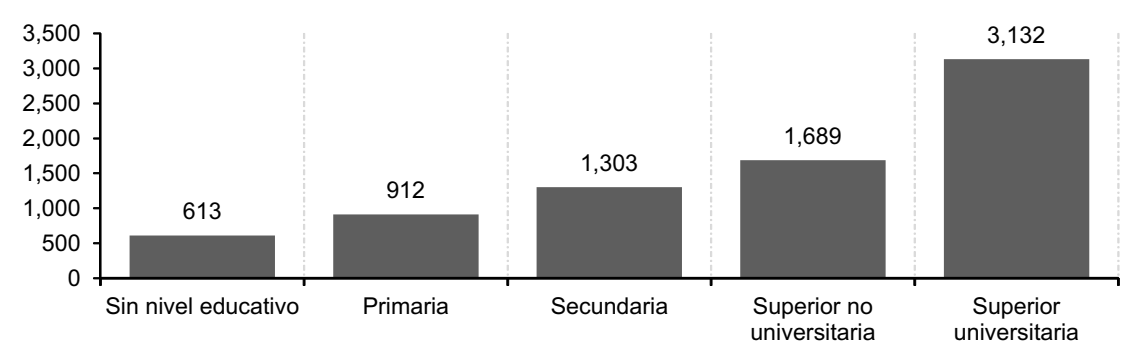

Fuente: INEI, 2016. Elaboración propia.

Gráfico 5. Perú: Asalariados e independientes por tipo de contrato según nivel educativo culminado, 2016

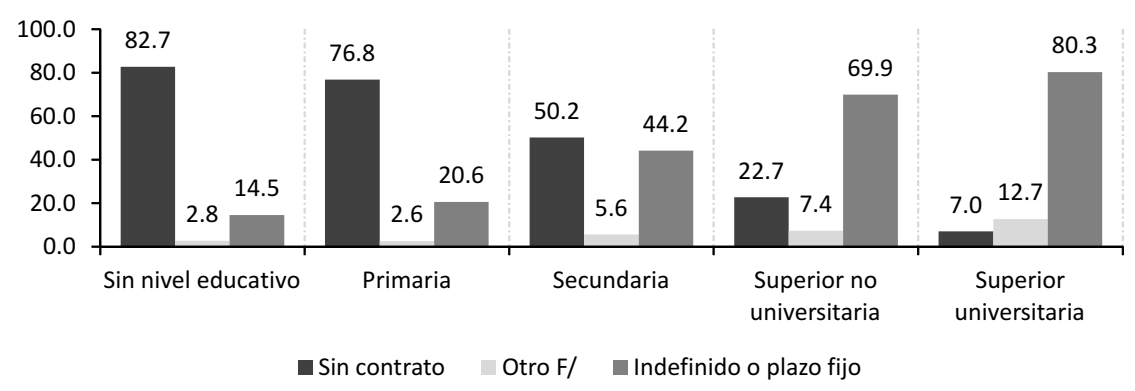

F/ Cifra referencial para sin nivel educativo.

Fuente: INEI, 2016. Elaboración propia. 
El Gráfico 6 no muestra grandes brechas ni una correlación aparente entre el nivel educativo culminado y el rango de horas trabajadas a la semana. Esto podría deberse a que este indicador toma mayor relevancia cuando se le analiza en conjunto con los demás indicadores: no es lo mismo trabajar menos de 48 horas con un ingreso igual a la remuneración mínima vital que trabajar menos de 48 horas ganando más de 3 veces la remuneración mínima vital (en este último caso, se obtiene 100 puntos en el indicador de ingresos).

Gráfico 6. Perú: Asalariados e independientes por rango de horas trabajadas a la semana según nivel educativo culminado, 2016

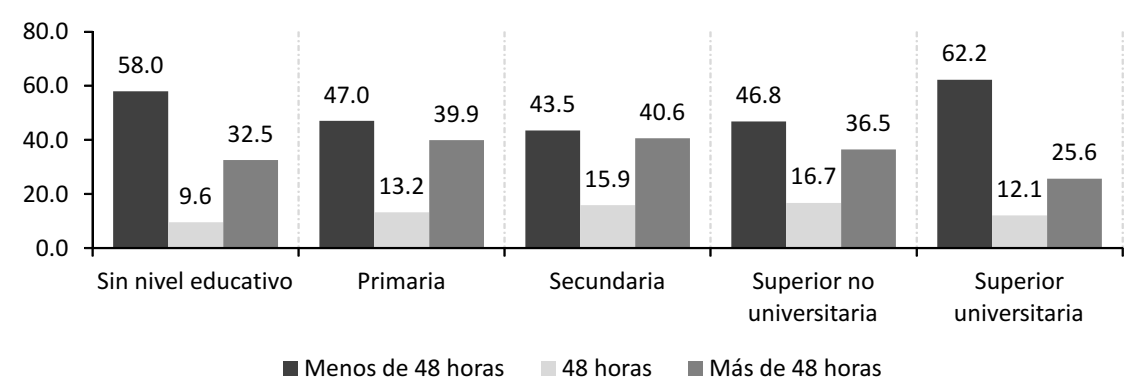

Fuente: INEI, 2016. Elaboración propia.

En resumen, según los gráficos mostrados anteriormente, un mayor nivel educativo culminado ha estado correlacionado positivamente con todos los indicadores del índice de calidad del empleo (ICE), a excepción del rango de horas trabajadas a la semana. Se esperaría, entonces, un efecto de causalidad positivo y significativo entre el ICE y el nivel educativo alcanzado al estimar el modelo econométrico.

Luego de haber detectado problemas de endogeneidad entre la variable educación y el ICE, se procedió a estimar un modelo de dos etapas siguiendo lo planteado en la metodología ${ }^{3}$.

3. Para poder cumplir con el objetivo, se usó el proceso condicional mixto (CMP por sus siglas en inglés) de David Roodman (2011). 
Tabla 5. Efectos marginales de pertenecer a un empleo de muy mala calidad

\begin{tabular}{|c|c|c|}
\hline Variable & $\mathrm{dy} / \mathrm{dx}$ & Std. \\
\hline Independiente ${ }^{\star * *}$ & 0.10059 & 0.00402 \\
\hline Educación ${ }^{* * *}$ & -0.1170641 & 0.00257 \\
\hline $\operatorname{Sexo}^{* * *}$ & -0.042953 & 0.00382 \\
\hline Área** & -0.0233524 & 0.00479 \\
\hline $\operatorname{Edad}^{\star * *}$ & -0.0233524 & 0.00057 \\
\hline $\operatorname{Edad}^{\wedge} 2^{* * *}$ & 0.0001723 & 0.00001 \\
\hline Industria & 0.0037438 & 0.00738 \\
\hline Construcción ${ }^{\star * *}$ & 0.0264855 & 0.00842 \\
\hline Comercio $^{* * *}$ & 0.1080596 & 0.00645 \\
\hline Servicios $^{\star * *}$ & -0.0277273 & 0.00533 \\
\hline \multicolumn{3}{|c|}{$\operatorname{Pr}(\mathrm{ICE}=1)=0,34389581$} \\
\hline
\end{tabular}

Fuente: Elaboración propia.

Las categorías bases $(\mathrm{x}=0)$ usadas para la estimación de los modelos son las siguientes: asalariado, mujer, rural y rama extractiva. Según los resultados obtenidos, todas las variables usadas como determinantes socioeconómicas fueron significativas al 95\% de confianza para cada categoría del índice de calidad del empleo, a excepción de la variable dummy industria.

En particular, según lo observado en la Tabla 5, la probabilidad promedio de tener un empleo de muy mala calidad es de $34.4 \%$. Con respecto a la educación, se encuentra una relación negativa: un mayor nivel educativo alcanzado redujo la probabilidad de tener un empleo de muy mala calidad en $11.7 \%$.

Por otro lado, un hombre tiene $4.2 \%$ menos de probabilidad promedio de tener un empleo de muy mala calidad en comparación con las mujeres. Cabe resaltar que, según lo mostrado en el Gráfico 7, para cada nivel educativo, se presentó una baja brecha de probabilidades entre hombres y mujeres: una mujer sin nivel educativo tiene una probabilidad de $81.2 \%$ de tener un empleo de muy mala calidad, mientras que un hombre tiene $77.9 \%$. De igual manera, una mujer con estudio superior universitario completo tiene una probabilidad de $4.9 \%$ de tener un empleo de muy mala calidad, mientras que un hombre del mismo nivel educativo presenta una probabilidad de $3.8 \%$. 
Gráfico 7. Perú: Probabilidad de pertenecer a un empleo de muy mala calidad por sexo, según nivel educativo alcanzado, 2016

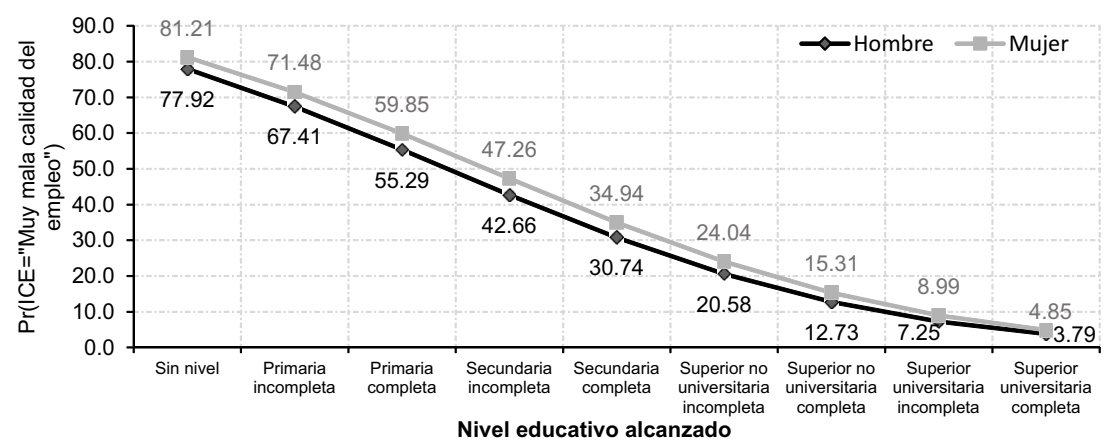

Fuente: Elaboración propia.

Gráfico 8. Perú: Probabilidad de pertenecer a un empleo de muy mala calidad por área, según nivel educativo alcanzado, 2016

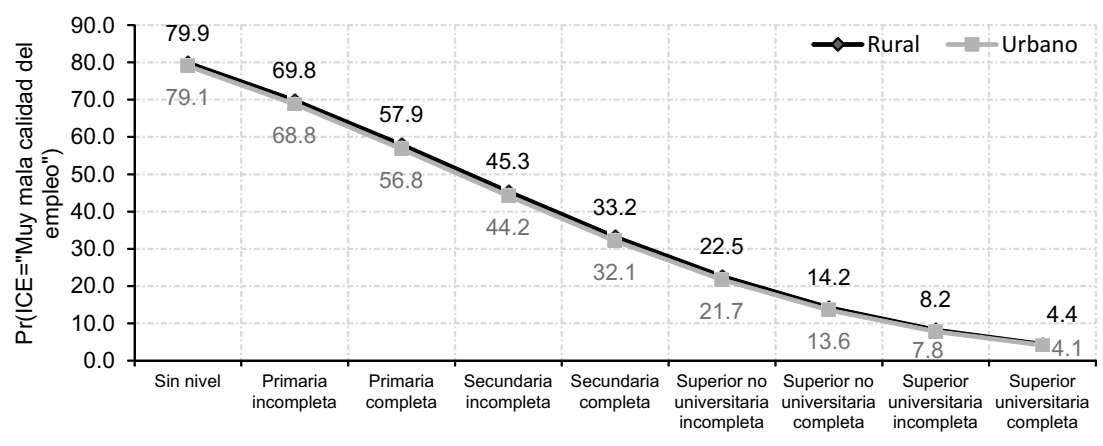

Nivel educativo alcanzado

Fuente: Elaboración propia.

Asimismo, según lo observado en el Gráfico 8, la brecha de tener un empleo de muy mala calidad entre la zona rural y urbana también es pequeña: la diferencia de probabilidades para cada nivel educativo de pertenecer a un empleo de muy mala calidad es de aproximadamente $1 \%$. Del mismo modo, una persona con secundaria completa tiene $40 \%$ menos de probabilidad de pertenecer a un empleo de muy mala calidad en comparación con las personas que no cuentan con ningún nivel. Además, en ambos casos, una persona sin nivel educativo tiene $70 \%$ más de probabilidades de tener un empleo de muy mala calidad en comparación de una persona con nivel educativo superior universitario completo. 
A diferencia de los dos grupos analizados anteriormente, en el Gráfico 9, se identifica que los independientes tienen mayores probabilidades de tener un empleo calificado como de muy mala calidad en comparación con los asalariados. La mayor brecha se presentó entre los trabajadores de primaria incompleta y secundaria completa. Además, los trabajadores con mayor educación alcanzado tuvieron muy pocas probabilidades de tener un empleo de muy mala calidad (5.5\% para los independientes y $3.1 \%$ para los asalariados).

Gráfico 9. Perú: Probabilidad de pertenecer a un empleo de muy mala calidad de independientes y asalariados, según nivel educativo alcanzado, 2016

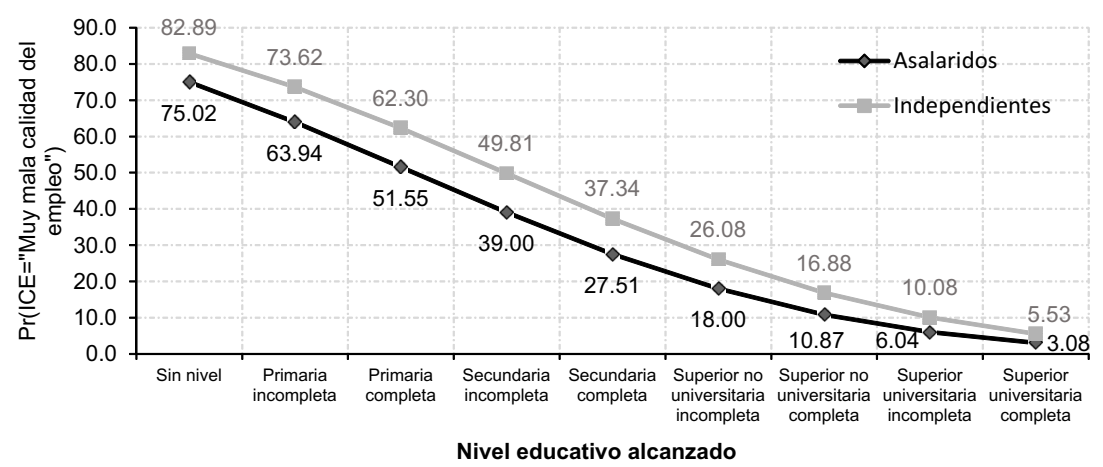

Fuente: Elaboración propia.

Tabla 6. Efectos marginales de pertenecer a un empleo de mala calidad

\begin{tabular}{lcc}
\hline \multicolumn{1}{c}{ Variable } & $\mathrm{dy} / \mathrm{dx}$ & Std. \\
\hline Independiente $^{* * *}$ & -0.0158066 & 0.00079 \\
Educación $^{* * *}$ & 0.0190638 & 0.00068 \\
Sexo $^{* *}$ & 0.0073891 & 0.00072 \\
Área $^{* *}$ & 0.0017576 & 0.00083 \\
Edad $^{* * *}$ & 0.0038029 & 0.00015 \\
Edad^2 $^{* * *}$ & -0.0000281 & 0.00000 \\
Industria $^{*}$ Construcción $^{* * *}$ & -0.0006247 & 0.00126 \\
Comercio $^{* * *}$ & -0.0050809 & 0.00186 \\
Servicios $^{* * *}$ & -0.0265144 & 0.00214 \\
\hline & 0.0042851 & 0.00079 \\
\hline
\end{tabular}

20 Fuente: Elaboración propia. 
En la Tabla 6, se muestra que, en promedio, la probabilidad de tener un empleo de mala calidad es de $42 \%$. Dicha probabilidad es la más alta entre las cuatro categorías de calidad laboral. Por el lado de la educación, un mayor nivel alcanzado redujo la probabilidad de pertenecer a un empleo de mala calidad en $1.9 \%$. Con respecto al género, un hombre tiene $0.7 \%$ menos de probabilidad de pertenecer a un empleo de mala calidad en comparación con una mujer.

En el Gráfico 10, se aprecia una relación no lineal entre la probabilidad de pertenecer a un empleo de mala calidad y el nivel educativo alcanzado por sexo. En el caso de los hombres, la educación alcanzada de los trabajadores que mayor probabilidad tuvo de tener un empleo de mala calidad fue secundaria completa, mientas que en el caso de las mujeres fue educación superior no universitaria incompleta.

Gráfico 10. Perú: Probabilidad de pertenecer a un empleo de mala calidad por sexo, según nivel educativo alcanzado, 2016

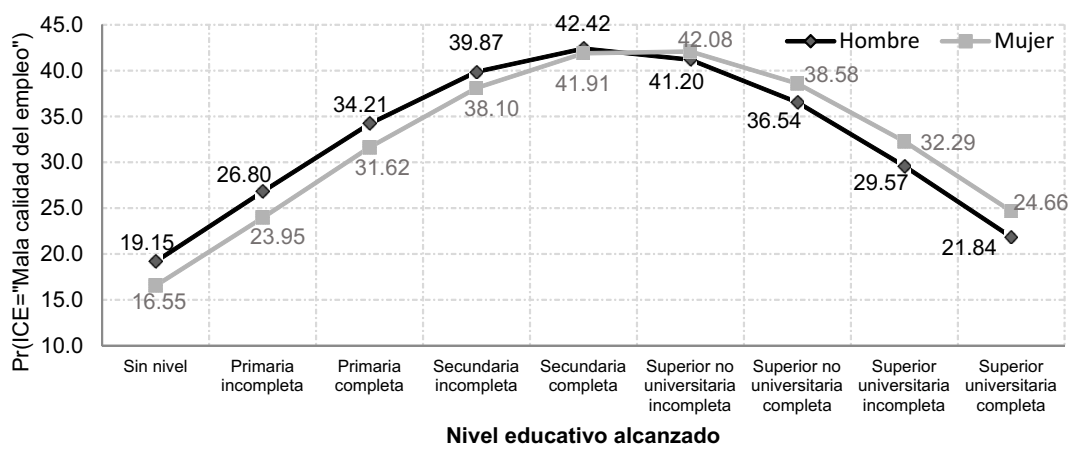

Fuente: Elaboración propia.

Por el lado de las brechas por áreas, no se encuentran diferencias significativas. En ambos casos, los trabajadores con secundaria completa son los que tienen la mayor probabilidad de pertenecer a un empleo de mala calidad.

En el análisis por categoría ocupacional, se encuentran brechas importantes en algunos niveles de educación. Por ejemplo, un trabajador independiente con nivel educativo superior universitario tiene $6,6 \%$ más de probabilidades de tener un empleo de mala calidad en comparación con un asalariado del mismo nivel. Además, un asalariado sin nivel educativo tiene $6.2 \%$ más de probabilidad de tener un empleo de mala calidad en comparación de un independiente sin nivel. Esto se debe a que los independientes sin nivel tienen mayor probabilidad de tener un empleo de muy mala calidad en comparación con los asalariados sin nivel (ver Gráfico 9). De este modo, se evidencia que los asalariados tienen una mayor probabilidad de tener un mejor empleo en comparación con los independientes. 
Gráfico 11. Perú: Probabilidad de pertenecer a un empleo de mala calidad por área, según nivel educativo alcanzado, 2016

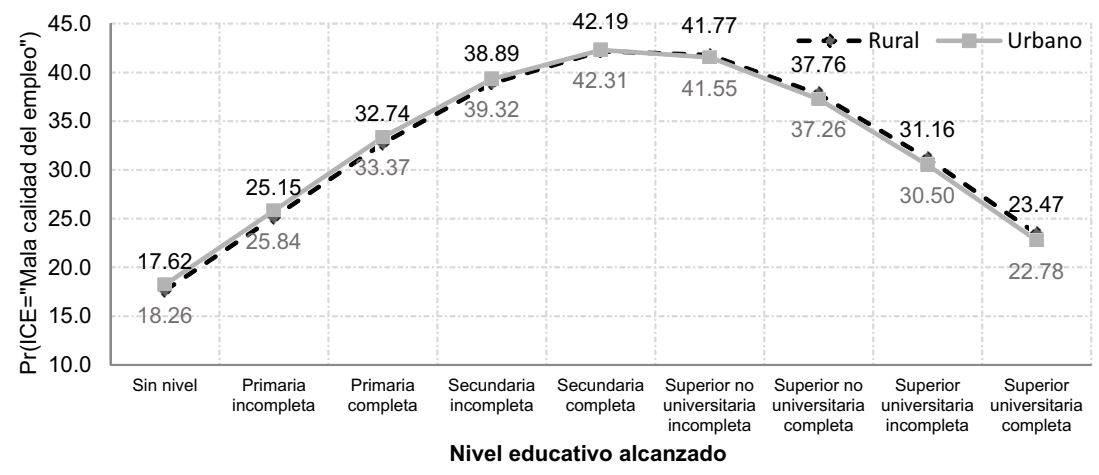

Fuente: Elaboración propia.

Gráfico 12. Perú: Probabilidad de pertenecer a un empleo de mala calidad de independientes y asalariados, según nivel educativo alcanzado, 2016

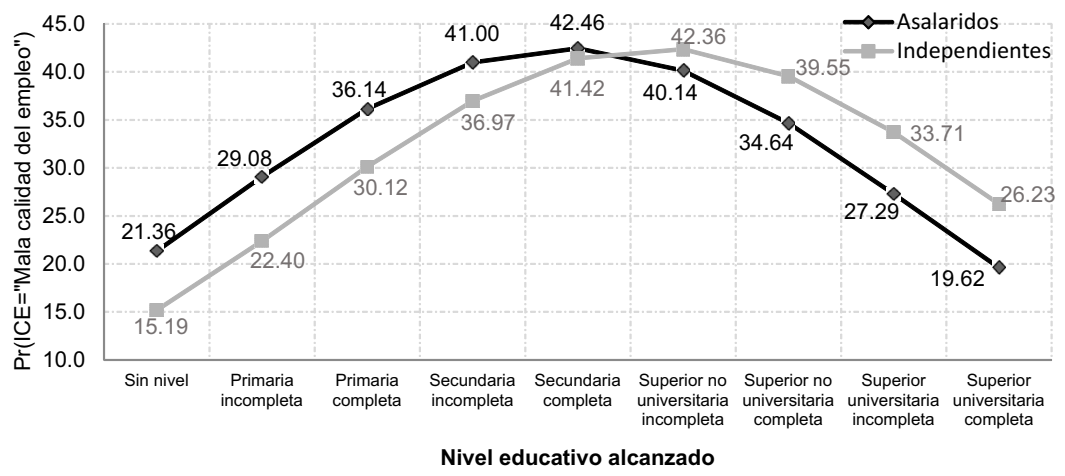

Fuente: Elaboración propia.

La Tabla 7 muestra que, en promedio, la probabilidad de pertenecer a un empleo de buena calidad es de aproximadamente 14\%. Además, un hombre tiene $1.6 \%$ más de probabilidad promedio de pertenecer a un empleo de buena calidad en comparación con una mujer. Asimismo, un mayor nivel educativo alcanzado aumenta la probabilidad de pertenecer a esta categoría en $4.4 \%$. 
Tabla 7. Efectos marginales de pertenecer a un empleo de buena calidad

\begin{tabular}{|c|c|c|}
\hline Variable & $\mathrm{dy} / \mathrm{dx}$ & Std. \\
\hline Independiente ${ }^{* * *}$ & -0.0377451 & 0.00162 \\
\hline Educación ${ }^{* * *}$ & 0.43993 & 0.00098 \\
\hline Sexo ${ }^{* * *}$ & 0.0160669 & 0.00143 \\
\hline Área ${ }^{* *}$ & 0.0039345 & 0.0018 \\
\hline $\operatorname{Edad}^{* * *}$ & 0.0087759 & 0.00024 \\
\hline $\operatorname{Edad}^{\wedge} 2^{* * *}$ & -0.0000648 & 0.00000 \\
\hline Industria & -0.0014045 & 0.00276 \\
\hline Construcción ${ }^{\star * *}$ & -0.0098224 & 0.00308 \\
\hline Comercio $^{* * *}$ & -0.0388436 & 0.00224 \\
\hline Servicios $^{* * *}$ & 0.0104531 & 0.00202 \\
\hline \multicolumn{3}{|c|}{$\operatorname{Pr}(\mathrm{ICE}=3)=0.14048684$} \\
\hline
\end{tabular}

Fuente: Elaboración propia.

Gráfico 13. Perú: Probabilidad de pertenecer a un empleo de buena calidad por sexo, según nivel educativo alcanzado, 2016

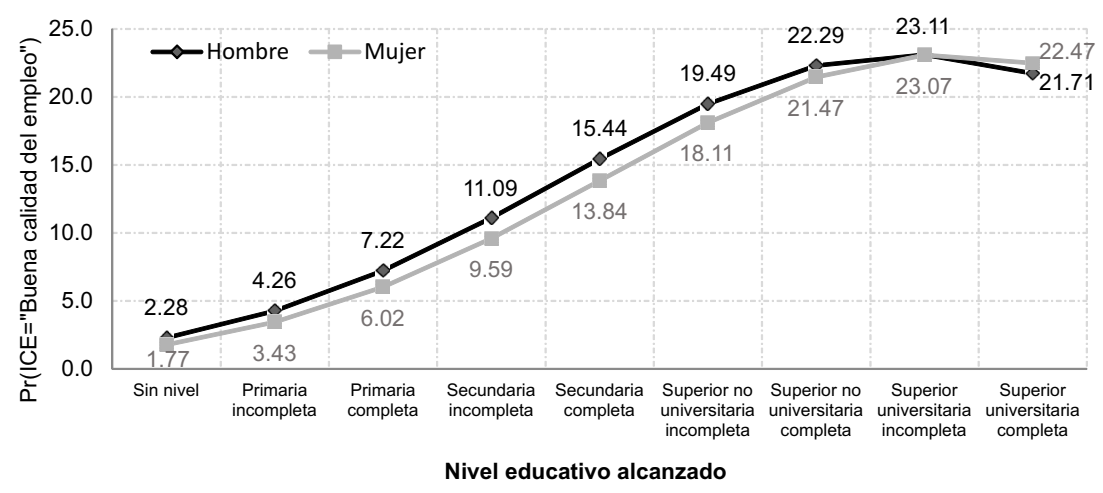

Fuente: Elaboración propia.

El Gráfico 13 muestra que, tanto para hombres como para mujeres, las personas con educación superior universitaria incompleta son las que tienen la mayor probabilidad de tener un empleo de buena calidad. Por el contrario, las personas sin nivel educativo tienen muy pocas probabilidades de tener un empleo de buena calidad (2.3\% para los hombres y $1.8 \%$ para las mujeres). 
Según el Gráfico 14, en el caso de las brechas por área, al igual que en el análisis por sexo, los trabajadores con educación superior universitaria incompleta fueron los que tuvieron la mayor probabilidad de pertenecer a este nivel de calidad del empleo. Además, las brechas de probabilidad son no significativas.

Otros resultados interesantes son los mostrados en el Gráfico 15, en que se observa que, para cada nivel educativo, los asalariados tuvieron mayor probabilidad de tener un empleo de buena calidad en comparación con los independientes, a excepción de los asalariados con niveles de educación superior universitaria. Esto último podría deberse a que los asalariados con nivel superior universitario tienen mayor probabilidad de pertenecer a una mejor categoría de calidad de empleo (muy buena calidad) en comparación con los independientes (ver Gráfico 18).

Gráfico 14. Perú: Probabilidad de pertenecer a un empleo de buena calidad por área, según nivel educativo alcanzado, 2016

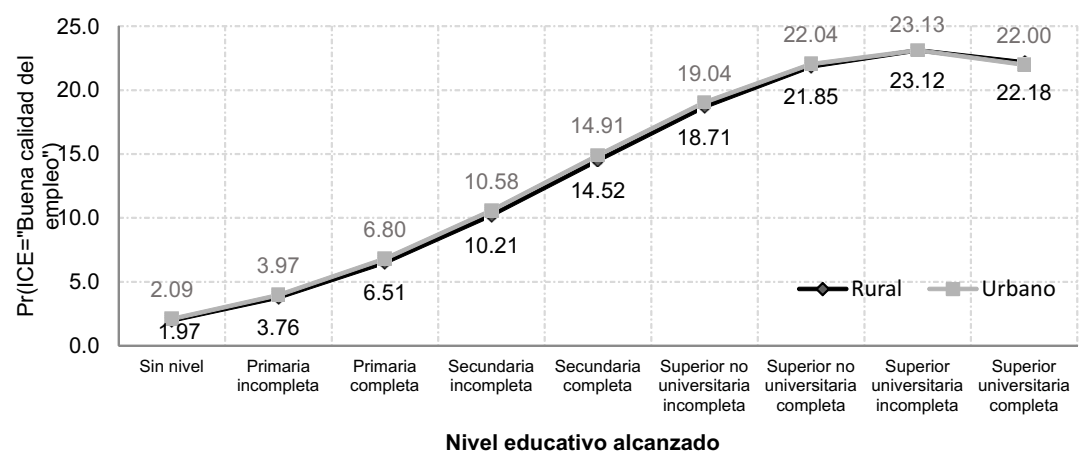

Fuente: Elaboración propia.

Gráfico 15. Perú: Probabilidad de pertenecer a un empleo de buena calidad por área, según nivel educativo alcanzado, 2016

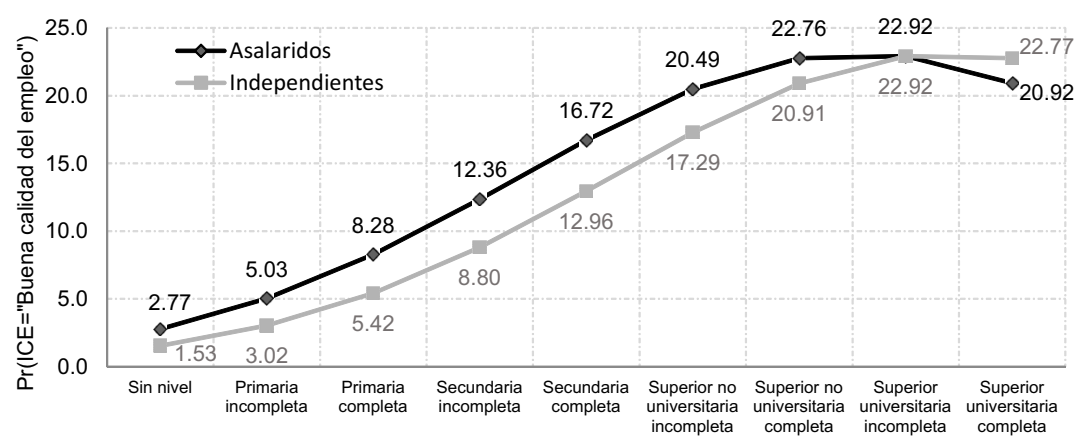

Fuente: Elaboración propia. 
Tabla 8. Efectos marginales de pertenecer a un empleo de muy buena calidad

\begin{tabular}{|c|c|c|}
\hline Variable & $\mathrm{dy} / \mathrm{dx}$ & Std. \\
\hline Independiente ${ }^{\star * *}$ & -0.0470383 & 0.0091 \\
\hline Educación ${ }^{* * *}$ & 0.0540073 & 0.00148 \\
\hline $\operatorname{Sexo}^{* * *}$ & 0.019497 & 0.00173 \\
\hline Área** & 0.0047989 & 0.00217 \\
\hline $\operatorname{Edad}^{\star * *}$ & 0.0107736 & 0.00028 \\
\hline $\operatorname{Edad}^{\wedge} 2^{* * *}$ & -0.0000795 & 0.00000 \\
\hline Industria & -0.0017146 & 0.00335 \\
\hline Construcción ${ }^{\star * *}$ & -0.0115923 & 0.00349 \\
\hline Comercio $^{* * *}$ & -0.0427015 & 0.00223 \\
\hline Servicios $^{* * *}$ & 0.012989 & 0.00254 \\
\hline \multicolumn{3}{|c|}{$\operatorname{Pr}(\mathrm{ICE}=4)=0,09557753$} \\
\hline
\end{tabular}

Fuente: Elaboración propia.

Finalmente, en la última estimación de efectos marginales mostrada en la Tabla 8 , se evidencia que, en promedio, la probabilidad de tener una muy buena calidad de empleo es de $9.6 \%$, cifra que se constituye como la menor probabilidad del índice de calidad. Asimismo, se observa que un mayor nivel educativo alcanzado aumenta la probabilidad de tener una muy buena calidad del empleo en $5.4 \%$. También, se detecta que un hombre tiene $1.9 \%$ más de probabilidad que una mujer de poseer un empleo de muy buena calidad y que un independiente tiene $4.7 \%$ menos de probabilidad de pertenecer a un empleo de muy buena calidad en comparación con los asalariados. Por último, una persona del área urbana tiene $0.5 \%$ más de probabilidad de acceder a un trabajo de muy buena calidad en comparación con una persona del área rural. 
Gráfico 16. Perú: Probabilidad de pertenecer a un empleo de muy buena calidad por sexo, según nivel educativo alcanzado, 2016

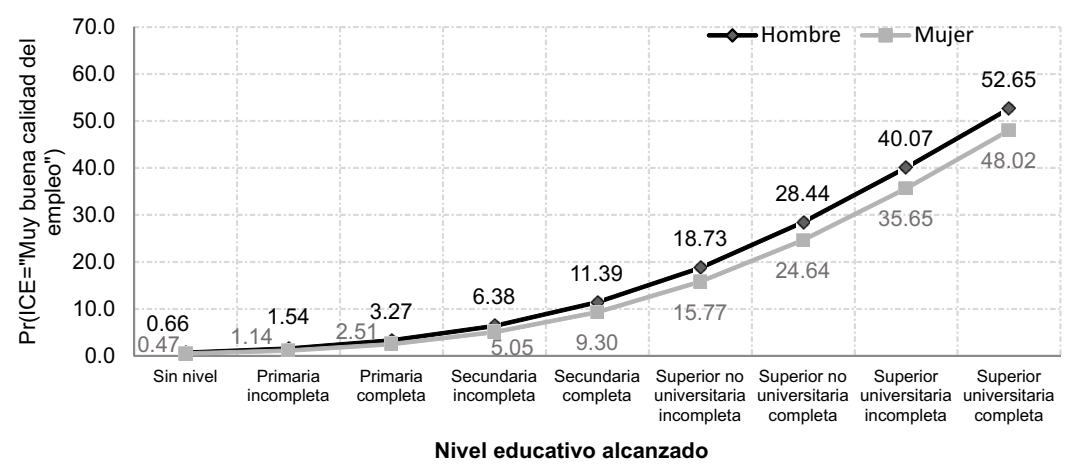

Fuente: Elaboración propia.

El Gráfico 16 muestra que la probabilidad de que una persona sin nivel educativo acceda a un empleo de muy buena calidad es muy limitada $(0.7 \%$ para los hombres y $0.5 \%$ para las mujeres). Lo mismo ocurre con los trabajadores con educación básica en general: ninguno supera el 12.0\% de probabilidades de acceder a la categoría de muy buena calidad de empleo.

Tanto en hombres (52.6\%) como en mujeres (48.0\%), los trabajadores con nivel educativo superior universitario completo fueron los que tuvieron mayor probabilidad de acceder a un empleo de muy buena calidad. No obstante, se debe anotar que, en este rubro, se presentó la mayor brecha de género en las probabilidades (4.6\%). Otro resultado importante es que un trabajador con estudio superior no universitario completo tiene casi la mitad de probabilidades (24.3\% para hombres y $23.4 \%$ para mujeres) de tener un empleo de muy buena calidad en comparación con los universitarios con estudios completos.

Con respecto al área, el Gráfico 14 muestra resultados similares a los mostrados anteriormente (brechas no significativas). Además, en concordancia con el análisis por sexo, la diferencia de probabilidades entre los trabajadores con nivel superior universitario completo y los trabajadores con educación superior no universitario incompleto es muy amplia (más de 20\% de diferencia). 
Gráfico 17. Perú: Probabilidad de pertenecer a un empleo de muy buena calidad por área, según nivel educativo alcanzado, 2016

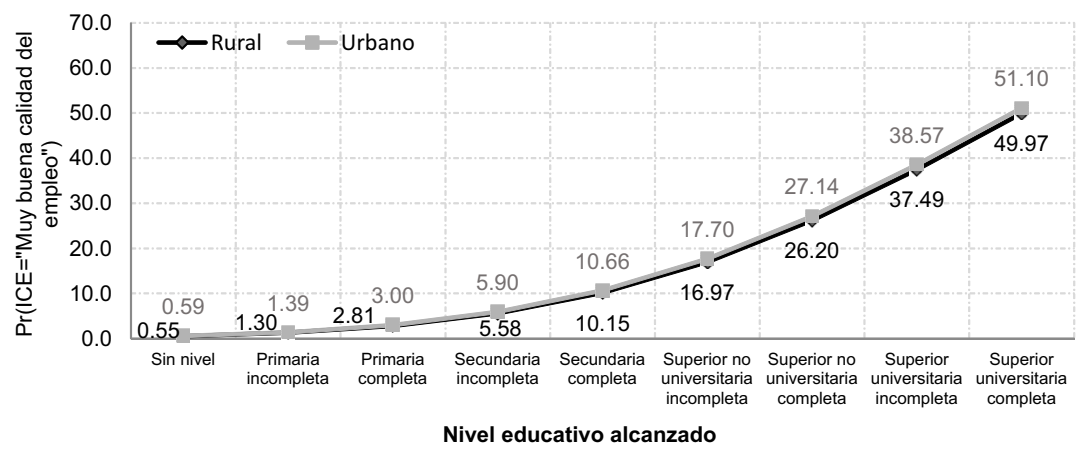

Fuente: Elaboración propia.

Por último, según categoría ocupacional, se identifica que un mayor nivel educativo alcanzado aumenta las probabilidades de acceder a un empleo de muy buena calidad. Sin embargo, a su vez, se observa que los beneficios de la educación han sido heterogéneos. La mayor brecha de probabilidades se dio en los trabajadores de mayor nivel (10.9\%).

Gráfico 18. Perú: Probabilidad de pertenecer a un empleo de muy buena calidad de independientes y asalariados, según nivel educativo alcanzado, 2016

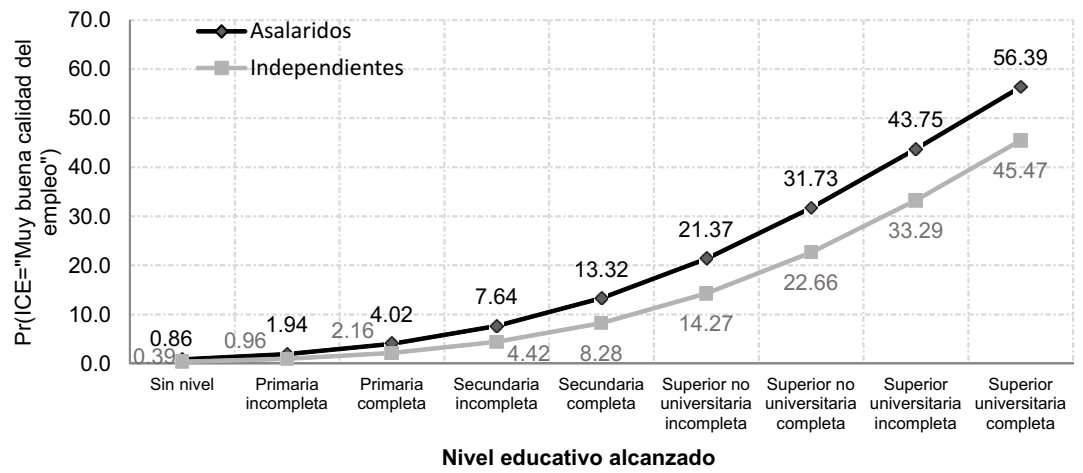

Fuente: Elaboración propia. 


\section{Conclusiones}

En líneas generales, se observa que la educación ha sido una variable importante para el acceso a una mejor calidad de empleo y que esta relación presenta no linealidades con respecto a algunas categorías del índice. En particular, el nivel educativo superior universitario completo mantiene grandes brechas de acceso a un empleo de muy buena calidad comparado con otros niveles educativos: un trabajador con estudio superior universitario completo tiene aproximadamente el doble de probabilidades de tener un empleo de muy buena calidad en comparación con un trabajador con estudio superior no universitario completo.

De igual manera, un empleado con estudio superior no universitario completo tiene aproximadamente tres veces más probabilidades de tener un empleo de muy buena calidad en comparación con un trabajador de secundaria completa. En contraste, trabajadores que tienen como grado alcanzado hasta secundaria completa presentan ínfimas probabilidades de tener un empleo de buena o muy buena calidad: los asalariados tienen $13.3 \%$ de tener un empleo de muy buena calidad y $16.7 \%$ de tener un empleo de buena calidad, mientras que los independientes tienen $8.3 \%$ y $13.0 \%$ para los mismos niveles de empleo, respectivamente.

Otro punto importante es que, aun en presencia de los mejores niveles educativos, se encuentran brechas tanto de género como de categoría ocupacional. Los hombres tienen mayor probabilidad de acceso a mejores índices de empleo para cada nivel educativo en comparación con las mujeres. Los mismo pasa entre asalariados e independientes, marco en que los asalariados son los más beneficiados. Es decir, el efecto de la educación ha sido diferente para algunos segmentos de la población. Con respecto a la ocupación, trabajadores de actividades económicas como construcción o comercio tienen menores probabilidades de tener un empleo de buena y muy buena calidad en comparación con la rama extractiva. Asimismo, la edad (proxy de experiencia) también aumenta la probabilidad de acceso a mejores índices de empleabilidad.

También, se debe recalcar que, si bien no se encontraron brechas significativas en el acceso a la buena calidad del empleo entre zonas urbanas y rurales, es recomendable velar por un acceso a la educación con igualdad de oportunidades sin importar el dominio geográfico, debido a que esta fue una variable significativa para medir el mayor nivel educativo de los trabajadores (ver Anexo 2).

En resumen, podemos concluir que la educación es una herramienta clave para acceder a una buena calidad de empleo, efectiva para reducir las probabilidades de tener empleos de muy mala calidad. Sin embargo, esta no ha sido suficiente para reducir las brechas presentes en género y categoría ocupacional; de hecho, las probabilidades han presentado considerables diferencias con respecto a estas características. Se recomienda, entonces, una política de incentivo a la educación superior universitaria y no universitaria, acompañada de una planificación social y económica que combata las brechas de género, así como las brechas de categoría de trabajo (asalariados, independientes o empleadores) y rama de actividad económica. 


\section{Referencias bibliográficas}

Alva, J. (2016). Determinantes de la calidad del empleo en Colombia para el periodo 2008-2014. Bogotá D.C.: Universidad de la Salle.

Barceinas, F. (2002). Endogeneidad y rendimientos de la educación. Ciudad de México: Universidad Autónoma Metropolitana.

Becker, G. S. (1964). Human capital: A theorical and empirical analysis, with special reference to education. New York: Columbia.

(1984). El capital humano: Un análisis teórico y empírico referido fundamentalmente a la educación. Madrid: Editorial Alianza.

Bustamante, C. D. y Arroyo, S. (2008). La raza como un determinante del acceso a un empleo de calidad: Un estudio para Cali. Ensayos sobre Política Económica, 26(57).

Cueto, B. y Pruneda, G. (2014). Nivel educativo y (des)empleo. Observatorio regional. Recuperado de: http://www.uniovi.net/regiolab/enlaces2/ observatorio/

Farné, S. (2003). Estudio sobre la calidad del empleo en Colombia. Resource document. Organización Internacional del Trabajo. Recuperado de http:// white.oit.org.pe/osra/documentos/farne_dic9.pdf

Freire, J. y Teijeiro, M. (2010). Las ecuaciones de Mincer y las tasas de rendimiento de la educación en Galicia. Investigaciones de Economía de la Educación (pp. 285-304), Vol. 5. Barcelona: Investigaciones de Economía de la Educación.

Gaviria, G. y Quingua, A. (2015). Determinantes de la calidad del empleo en la ciudad de Cali en 2012. Magazín Empresarial, 11(25), 11-21.

Gonzales, C. y Mora, J. (2011). Políticas activas para la creación de empleo digno y de calidad en la Ciudad de Cali-Colombia. Estudios Gerenciales, 27(118).

Hanushek, E. (2008). Education production functions. In S. N. Durlauf y L. E. Blume (Eds.), The New Palgrave Dictionary of Economics, 24(3), 11411177.

Instituto Nacional de Estadística e Informática [INEI] (2016). Encuesta Nacional de Hogares sobre Condiciones de Vida y Pobreza. Recuperado de: http://iinei.inei.gob.pe/microdatos/

Ministerio de Trabajo y Promoción del Empleo [MTPE] (2016). Informe Anual del Empleo en el Perú, 2016. Lima: MTPE. Recuperado de http:// www.trabajo.gob.pe/archivos/file/estadisticas/peel/enaho/INFORME_ ANUAL_EMPLEO_ENAHO_2016.pdf

Mora, J. y Ulloa, M. (2011). El efecto de la educación sobre la calidad del empleo en Colombia. Borradores de Economía y Finanzas, 1(26). 
Pastor, J., J. Raymond, J. Roig y L. Serrano (2008) El rendimiento del capital humano en España, Bancaja, Valencia, 2007. Valencia. Instituto Valenciano de Investigaciones Económicas, Ivie.

Posso, C. M. (2010). Calidad del empleo y segmentación laboral: Un análisis para el mercado laboral colombiano 2001-2006. Desarrollo y Sociedad, 65, 191-234.

Riomaña, O. (2011). Incidencia del nivel educativo en el proceso de búsqueda de empleo y en la duración del desempleo en Colombia: Un análisis desde la teoría de la señalización. Cali: Universidad del Valle-Cidse.

Rivera, T. (2017). Determinantes del nivel educativo alcanzado. Un análisis multinivel para Cali. Santiago de Cali. Universidad del Valle.

Rodríguez, C.y J. Cáceres (2007). Modelos de elección discreta y especificaciones ordenada: Una reflexión metodológica. Estadística Española, 49(166), 451-471.

Roodman, D. (2011). Estimating fully observed recursive mixed-process models with cmp. Stata Journal, 11(2), 159-206.

Rosenthal, N. (1989). More than wages at issue in job quality debate. Monthly Labor Review, diciembre.

Schultz, T. (1961). Investment in human capital. The American Economic Review 51(1), 1-17.

Spence, M. (2002). Signaling in retrospect and the informational structure of markets. American Economic Review 92(3), 434-59.

Valenzuela, M. E. (2000). La calidad del empleo de las mujeres en los países del Cono Sur. En M. E. Valenzuela y G. Reinecke, G. (Eds.), ¿Más y mejores empleos para las mujeres? La experiencia de los países del Mercosur y Chile. Santiago de Chile: Organización Internacional del Trabajo.

Verdera. F. (1995). Propuestas de redefinición de la medición del subempleo y el desempleo y de nuevos indicadores sobre la situación ocupacional en Lima (documento de trabajo No. 22). Organización Internacional del Trabajo, Lima.

Villarreal E. (2018). Endogeneidad de los rendimientos educativos en México. Perfiles Latinoamericanos, 26(51), 265-299. Recuperado de: https://doi. org/10.18504/pl2651-011-2018.

Weller, J. y Roethlisberger, C. (2011). La calidad del empleo en América Latina. Santiago de Chile: División de Desarrollo Económico - Cepal. 


\section{Anexos}

Anexo 1. Estimación del modelo probit ordenado del índice de calidad del empleo sin considerar endogenedidad

\begin{tabular}{|c|c|c|}
\hline Variable & $\mathrm{dy} / \mathrm{dx}$ & Std. \\
\hline Independiente ${ }^{\star * *}$ & -0.29418 & 0.01109 \\
\hline Educación ${ }^{\star * *}$ & 0.243707 & 0.00267 \\
\hline Sexo ${ }^{* * *}$ & 0.090438 & 0.01025 \\
\hline Área ${ }^{* *}$ & 0.059002 & 0.01288 \\
\hline $\operatorname{Edad}^{* * *}$ & 0.061319 & 0.00157 \\
\hline $\operatorname{Edad} 2^{\wedge * *}$ & -0.00044 & 0.00002 \\
\hline Industria & 0.007514 & 0.02024 \\
\hline Construcción ${ }^{\star * *}$ & -0.06636 & 0.02273 \\
\hline Comercio $^{* * *}$ & -0.27693 & 0.01688 \\
\hline Servicios ${ }^{* * *}$ & 0.087261 & 0.01480 \\
\hline Cut points 1 & 2.40483 & 0.03750 \\
\hline Cut points 2 & 3.542837 & 0.03859 \\
\hline Cut points 3 & 4.1396 & 0.03953 \\
\hline Log likelihood & \multicolumn{2}{|c|}{$-63,431.2140$} \\
\hline LR chi2(10) & \multicolumn{2}{|c|}{$17,590.0000$} \\
\hline $\mathrm{N}$ & \multicolumn{2}{|c|}{57,117} \\
\hline
\end{tabular}

Fuente: Elaboración propia. 
Anexo 2. Estimación del modelo probit ordenado de la educación

\begin{tabular}{|c|c|c|}
\hline Variable & Coef. & Std. \\
\hline $\operatorname{Jefe}^{\star * *}$ & -0.2447 & 0.0089 \\
\hline Casado $^{* * *}$ & -0.1634 & 0.0092 \\
\hline Miembros $(<13 \text { años })^{* * *}$ & 0.0311 & 0.0042 \\
\hline Selva ${ }^{* *}$ & -0.7669 & 0.0158 \\
\hline Costa norte $e^{* * *}$ & -0.5516 & 0.0165 \\
\hline Costa centro ${ }^{\star * *}$ & -0.3066 & 0.0179 \\
\hline Costa sur ${ }^{\star * *}$ & -0.2635 & 0.0192 \\
\hline Sierra norte ${ }^{\star * *}$ & -0.9980 & 0.0207 \\
\hline Sierra centro ${ }^{* * *}$ & -0.8084 & 0.0164 \\
\hline Sierra sur ${ }^{* * *}$ & -0.6790 & 0.0173 \\
\hline Cut points 1 & -2.5559 & 0.0170 \\
\hline Cut points 2 & -1.7233 & 0.01528 \\
\hline Cut points 3 & -1.2967 & 0.0148 \\
\hline Cut points 4 & -0.9191 & 0.0145 \\
\hline Cut points 5 & -0.2298 & 0.0141 \\
\hline Cut points 6 & -0.0967 & 0.0141 \\
\hline Cut points 7 & 0.2516 & 0.0142 \\
\hline Cut points 8 & 0.5083 & 0.0144 \\
\hline Log likelihood & \multicolumn{2}{|c|}{$-115,315.9000$} \\
\hline LR chi2(10) & \multicolumn{2}{|c|}{$5,940.2400$} \\
\hline $\mathrm{N}$ & \multicolumn{2}{|c|}{57,522} \\
\hline
\end{tabular}

Fuente: Elaboración propia. 
Anexo 3. Estimación del modelo probit ordenado del índice de calidad del empleo con corrección de endogeneidad de la variable educación

\begin{tabular}{|c|c|c|}
\hline Variable & Coef. & Std. \\
\hline \multicolumn{3}{|l|}{ ICE } \\
\hline Independiente $e^{* *}$ & -0.27463 & 0.01111 \\
\hline Educación $^{* * *}$ & 0.318112 & 0.00685 \\
\hline Sexo ${ }^{* * *}$ & 0.116313 & 0.11631 \\
\hline Área** & 0.028452 & 0.02845 \\
\hline $\operatorname{Edad}^{* * *}$ & 0.063458 & 0.06346 \\
\hline $\operatorname{Edad}^{\wedge} 2^{* * *}$ & -0.00047 & -0.00047 \\
\hline Industria & -0.01016 & -0.01016 \\
\hline Construcción ${ }^{* * *}$ & -0.07111 & -0.00711 \\
\hline Comercio $^{\star * *}$ & -0.28485 & -0.28485 \\
\hline Servicios $^{\star * *}$ & 0.0756 & 0.07563 \\
\hline \multicolumn{3}{|l|}{ Educación } \\
\hline $\mathrm{Jefe}^{* * *}$ & -0.22831 & 0.00899 \\
\hline Casado ${ }^{* * *}$ & -0.13696 & 0.00949 \\
\hline Miembros $(<13 \text { años })^{* * *}$ & 0.039726 & 0.00422 \\
\hline Selva ${ }^{\star *}$ & -0.78868 & 0.01556 \\
\hline Costa norte & -0.57969 & 0.01636 \\
\hline Costa centro & -0.34096 & 0.01783 \\
\hline Costa sur*** & -0.30453 & 0.01919 \\
\hline Sierra norte & -1.0059 & 0.02049 \\
\hline Sierra centro ${ }^{\star * *}$ & -0.83488 & 0.01625 \\
\hline Sierra sur ${ }^{* * *}$ & -0.7052 & 0.01713 \\
\hline Cut points $1^{\star * *}$ & 2.8062 & 0.04873 \\
\hline Cut points $2^{* * *}$ & 3.9271 & 0.04755 \\
\hline Cut points $3^{* * *}$ & 4.5152 & 0.04733 \\
\hline Cut points $1^{* * *}$ & -2.55566 & 0.01696 \\
\hline Cut points $2^{\star * *}$ & -1.71272 & 0.15350 \\
\hline Cut points $3^{* * *}$ & -1.2821 & 0.01492 \\
\hline Cut points $4^{\star * *}$ & -0.9031 & 0.01460 \\
\hline Cut points $5^{\star \star *}$ & -0.2164 & 0.01422 \\
\hline Cut points $6^{* * *}$ & -0.0846 & 0.01420 \\
\hline Cut points $7^{\star \star *}$ & 0.261252 & 0.01420 \\
\hline Cut points $8^{* * *}$ & 0.5150 & 0.01444 \\
\hline Log likelihood & \multicolumn{2}{|c|}{$-178,687.9600$} \\
\hline LR chi2(10) & \multicolumn{2}{|c|}{$12,741.7800$} \\
\hline $\mathrm{N}$ & \multicolumn{2}{|c|}{57,522} \\
\hline
\end{tabular}

Fuente: Elaboración propia. 
Anexo 4. Probabilidades de pertenecer a un empleo de muy mala calidad

\begin{tabular}{|c|c|c|c|c|c|c|}
\hline Nivel educativo alcanzado & Hombre & Std. & Mujer & Std. & Rural & Std. \\
\hline Sin nivel & 77.91508 & 0.78573 & 81.20947 & 0.81289 & 79.86201 & 0.75297 \\
\hline Primaria incompleta & 67.40832 & 0.76969 & 71.48228 & 0.75135 & 69.79768 & 0.71884 \\
\hline Primaria completa & 55.29446 & 0.63703 & 59.84811 & 0.57722 & 57.94467 & 0.58805 \\
\hline Secundaria incompleta & 42.66115 & 0.44121 & 47.26164 & 0.36215 & 45.31789 & 0.43654 \\
\hline Secundaria completa & 30.744 & 0.30601 & 34.94497 & 0.25188 & 33.15105 & 0.3814 \\
\hline $\begin{array}{l}\text { Superior no universitaria } \\
\text { incompleta }\end{array}$ & 20.57571 & 0.32287 & 24.04304 & 0.30041 & 22.54672 & 0.41768 \\
\hline $\begin{array}{l}\text { Superior no universitaria } \\
\text { completa }\end{array}$ & 12.72799 & 0.36689 & 15.31467 & 0.33945 & 14.18667 & 0.43222 \\
\hline $\begin{array}{l}\text { Superior universitaria } \\
\text { incompleta }\end{array}$ & 7.24953 & 0.35111 & 8.99371 & 0.31237 & 8.2252 & 0.38441 \\
\hline \multirow{2}{*}{$\begin{array}{l}\text { Superior universitaria } \\
\text { completa }\end{array}$} & 3.79021 & 0.28288 & 4.85322 & 3.79021 & 4.38002 & 0.29414 \\
\hline & Urbano & Std. & $\begin{array}{l}\text { Asala- } \\
\text { riados }\end{array}$ & Std. & $\begin{array}{l}\text { Indepen- } \\
\text { dientes }\end{array}$ & Std. \\
\hline Sin nivel & 79.05267 & 0.83577 & 75.01754 & 0.94149 & 82.886 & 0.68229 \\
\hline Primaria incompleta & 68.79819 & 0.79442 & 63.94278 & 0.86627 & 73.61621 & 0.66498 \\
\hline Primaria completa & 56.82915 & 0.63243 & 51.54822 & 0.67486 & 62.3029 & 0.53871 \\
\hline Secundaria incompleta & 44.19267 & 0.40903 & 39.00095 & 0.43998 & 49.81385 & 0.3617 \\
\hline Secundaria completa & 32.12529 & 0.25281 & 27.51181 & 0.27983 & 37.34316 & 0.28623 \\
\hline $\begin{array}{l}\text { Superior no universitaria } \\
\text { incompleta }\end{array}$ & 21.70161 & 0.27263 & 17.99598 & 0.26626 & 26.0797 & 0.36459 \\
\hline $\begin{array}{l}\text { Superior no universitaria } \\
\text { completa }\end{array}$ & 13.55741 & 0.32027 & 10.86703 & 0.28242 & 16.87788 & 0.42794 \\
\hline $\begin{array}{l}\text { Superior universitaria } \\
\text { incompleta }\end{array}$ & 7.80174 & 0.30612 & 6.03619 & 0.2539 & 10.07809 & 0.41213 \\
\hline $\begin{array}{l}\text { Superior universitaria } \\
\text { completa }\end{array}$ & 4.12248 & 0.24319 & 3.0752 & 0.19202 & 5.53308 & 0.33451 \\
\hline
\end{tabular}

Fuente: Elaboración propia. 
Anexo 5. Probabilidades de pertenecer a un empleo de mala calidad

\begin{tabular}{|c|c|c|c|c|c|c|}
\hline Nivel educativo alcanzado & Hombre & Std. & Mujer & Std. & Rural & Std. \\
\hline Sin nivel & 19.14837 & 0.65287 & 16.55047 & 0.64827 & 17.624 & 0.61517 \\
\hline Primaria incompleta & 26.7952 & 0.55444 & 23.95103 & 0.5876 & 25.14649 & 0.54207 \\
\hline Primaria completa & 34.21304 & 0.39685 & 31.62209 & 44.683 & 32.73612 & 0.40686 \\
\hline Secundaria incompleta & 39.87174 & 0.27939 & 38.10249 & 0.30964 & 38.89485 & 0.29173 \\
\hline Secundaria completa & 42.41911 & 0.25421 & 41.90963 & 0.25442 & 41.77291 & 0.30239 \\
\hline $\begin{array}{l}\text { Superior no universitaria } \\
\text { incompleta }\end{array}$ & 41.20177 & 0.30351 & 42.08481 & 0.27762 & 41.77291 & 0.30239 \\
\hline $\begin{array}{l}\text { Superior no universitaria } \\
\text { completa }\end{array}$ & 36.53519 & 0.45939 & 38.58248 & 0.40861 & 37.76352 & 0.48159 \\
\hline $\begin{array}{l}\text { Superior universitaria } \\
\text { incompleta }\end{array}$ & 29.57206 & 0.65897 & 32.28937 & 0.61591 & 31.16326 & 0.70825 \\
\hline \multirow{2}{*}{$\begin{array}{l}\text { Superior universitaria } \\
\text { completa }\end{array}$} & 21.84302 & 0.79223 & 24.66247 & 0.78601 & 23.4694 & 0.86587 \\
\hline & Urbano & Std. & $\begin{array}{l}\text { Asala- } \\
\text { riados }\end{array}$ & Std. & $\begin{array}{l}\text { Indepen- } \\
\text { dientes }\end{array}$ & Std. \\
\hline Sin nivel & 18.26155 & 0.67678 & 21.35706 & 0.73339 & 15.19392 & 0.57236 \\
\hline Primaria incompleta & 25.84295 & 0.59109 & 29.0842 & 0.60835 & 22.39881 & 0.52182 \\
\hline Primaria completa & 33.36856 & 0.43235 & 36.14306 & 0.42512 & 30.12304 & 0.39808 \\
\hline Secundaria incompleta & 39.32404 & 0.2965 & 40.99788 & 0.28949 & 36.96948 & 0.28253 \\
\hline Secundaria completa & 42.30523 & 0.25487 & 42.45574 & 0.25748 & 41.41608 & 0.24501 \\
\hline $\begin{array}{l}\text { Superior no universitaria } \\
\text { incompleta }\end{array}$ & 41.55145 & 0.28733 & 40.13929 & 0.30822 & 42.35834 & 0.27102 \\
\hline $\begin{array}{l}\text { Superior no universitaria } \\
\text { completa }\end{array}$ & 37.25842 & 0.42104 & 34.64413 & 0.45296 & 39.55176 & 0.40999 \\
\hline $\begin{array}{l}\text { Superior universitaria } \\
\text { incompleta }\end{array}$ & 30.49636 & 0.61544 & 27.29199 & 0.62388 & 33.71406 & 0.64024 \\
\hline $\begin{array}{l}\text { Superior universitaria } \\
\text { completa }\end{array}$ & 22.77974 & 0.76037 & 19.61823 & 0.72362 & 26.22933 & 0.83879 \\
\hline
\end{tabular}

Fuente: Elaboración propia. 
Anexo 6. Probabilidades de pertenecer a un empleo de buena calidad

\begin{tabular}{|c|c|c|c|c|c|c|}
\hline Nivel educativo alcanzado & Hombre & Std. & Mujer & Std. & Rural & Std. \\
\hline Sin nivel & 2.27659 & 0.12487 & 1.76665 & 0.10865 & 1.96874 & 0.10909 \\
\hline Primaria incompleta & 4.25878 & 0.15296 & 3.42605 & 0.14151 & 3.76052 & 0.1391 \\
\hline Primaria completa & 7.21968 & 0.15886 & 6.02089 & 0.15671 & 6.50934 & 0.15496 \\
\hline Secundaria incompleta & 11.09188 & 0.15543 & 9.58903 & 0.15747 & 10.21117 & 0.16884 \\
\hline Secundaria completa & 15.44415 & 0.18313 & 13.8406 & 0.17783 & 14.51719 & 0.20729 \\
\hline $\begin{array}{l}\text { Superior no universitaria } \\
\text { incompleta }\end{array}$ & 19.48988 & 0.2302 & 18.10575 & 0.22502 & 18.70567 & 0.25273 \\
\hline $\begin{array}{l}\text { Superior no universitaria } \\
\text { completa }\end{array}$ & 22.29207 & 0.24823 & 21.46697 & 0.25123 & 21.84531 & 0.26035 \\
\hline $\begin{array}{l}\text { Superior universitaria } \\
\text { incompleta }\end{array}$ & 23.10965 & 0.26673 & 23.06882 & 0.2528 & 23.123 & 0.25674 \\
\hline \multirow{2}{*}{$\begin{array}{l}\text { Superior universitaria } \\
\text { completa }\end{array}$} & 21.71399 & 0.38511 & 22.46897 & 0.33288 & 22.18367 & 0.36814 \\
\hline & Urbano & Std. & $\begin{array}{l}\text { Asala- } \\
\text { riados }\end{array}$ & Std. & $\begin{array}{l}\text { Indepen- } \\
\text { dientes }\end{array}$ & Std. \\
\hline Sin nivel & 2.09444 & 0.12402 & 2.76927 & 0.15812 & 12.52785 & 0.08857 \\
\hline Primaria incompleta & 3.96554 & 0.1561 & 5.03138 & 0.19191 & 3.0223 & 0.11513 \\
\hline Primaria completa & 6.80403 & 0.16594 & 8.28415 & 0.19941 & 5.41766 & 0.12634 \\
\hline Secundaria incompleta & 10.57992 & 0.15937 & 12.36144 & 0.19139 & 8.80095 & 0.12982 \\
\hline Secundaria completa & 14.90969 & 0.17622 & 16.71732 & 0.20479 & 12.95716 & 0.16433 \\
\hline $\begin{array}{l}\text { Superior no universitaria } \\
\text { incompleta }\end{array}$ & 19.04317 & 0.22163 & 20.49056 & 0.23664 & 17.28895 & 0.22576 \\
\hline $\begin{array}{l}\text { Superior no universitaria } \\
\text { completa }\end{array}$ & 22.04473 & 0.24632 & 22.7635 & 0.25029 & 20.90827 & 0.25768 \\
\hline $\begin{array}{l}\text { Superior universitaria } \\
\text { incompleta }\end{array}$ & 23.1298 & 0.26047 & 22.92065 & 0.28271 & 22.91747 & 0.24954 \\
\hline $\begin{array}{l}\text { Superior universitaria } \\
\text { completa }\end{array}$ & 21.99593 & 0.36041 & 20.91789 & 0.40496 & 22.76765 & 0.3157 \\
\hline
\end{tabular}

Fuente: Elaboración propia. 
Anexo 7. Probabilidades de pertenecer a un empleo de buena calidad

\begin{tabular}{|c|c|c|c|c|c|c|}
\hline Nivel educativo alcanzado & Hombre & Std. & Mujer & Std. & Rural & Std. \\
\hline Sin nivel & 0.65995 & 0.03611 & 0.47342 & 0.04533 & 0.54525 & 0.03772 \\
\hline Primaria incompleta & 1.5377 & 0.05996 & 1.14065 & 0.0709 & 1.29531 & 0.0617 \\
\hline Primaria completa & 3.27282 & 0.08518 & 2.50891 & 0.09401 & 2.80987 & 0.08981 \\
\hline Secundaria incompleta & 6.37524 & 0.11084 & 5.04684 & 0.11755 & 5.57609 & 0.13333 \\
\hline Secundaria completa & 11.39273 & 0.17484 & 9.30479 & 0.19827 & 10.14598 & 0.23847 \\
\hline $\begin{array}{l}\text { Superior no universitaria } \\
\text { incompleta }\end{array}$ & 18.73264 & 0.34144 & 15.7664 & 0.39609 & 16.9747 & 0.4486 \\
\hline $\begin{array}{l}\text { Superior no universitaria } \\
\text { completa }\end{array}$ & 28.44475 & 0.61872 & 24.63588 & 0.69657 & 26.2045 & 0.75879 \\
\hline $\begin{array}{l}\text { Superior universitaria } \\
\text { incompleta }\end{array}$ & 40.06877 & 0.96021 & 35.6481 & 1.03755 & 37.48855 & 1.11495 \\
\hline \multirow{2}{*}{$\begin{array}{l}\text { Superior universitaria } \\
\text { completa }\end{array}$} & 52.62578 & 1.28209 & 48.01533 & 1.32818 & 49.96691 & 1.42756 \\
\hline & Urbano & Std. & $\begin{array}{l}\text { Asala- } \\
\text { riados }\end{array}$ & Std. & $\begin{array}{c}\text { Indepen- } \\
\text { dientes }\end{array}$ & Std. \\
\hline Sin nivel & 0.59133 & 0.04371 & 0.85613 & 0.06155 & 0.39222 & 0.02821 \\
\hline Primaria incompleta & 1.39332 & 0.0702 & 1.94464 & 0.09538 & 0.96269 & 0.04696 \\
\hline Primaria completa & 2.99826 & 0.09518 & 4.02457 & 0.12556 & 2.15639 & 0.06729 \\
\hline Secundaria incompleta & 5.90336 & 0.11495 & 7.63974 & 0.14795 & 4.41572 & 0.09436 \\
\hline Secundaria completa & 10.6598 & 0.17346 & 13.31514 & 0.21084 & 8.2836 & 0.1728 \\
\hline $\begin{array}{l}\text { Superior no universitaria } \\
\text { incompleta }\end{array}$ & 17.70377 & 0.34493 & 21.37418 & 0.39056 & 14.27302 & 0.3552 \\
\hline $\begin{array}{l}\text { Superior no universitaria } \\
\text { completa }\end{array}$ & 27.13944 & 0.62699 & 31.72534 & 0.67377 & 22.6621 & 0.64718 \\
\hline $\begin{array}{l}\text { Superior universitaria } \\
\text { incompleta }\end{array}$ & 38.5721 & 0.96219 & 43.75117 & 0.98834 & 33.29037 & 10.0816 \\
\hline $\begin{array}{l}\text { Superior universitaria } \\
\text { completa }\end{array}$ & 51.10185 & 1.26235 & 56.38868 & 1.24197 & 45.46994 & 1.35645 \\
\hline
\end{tabular}

Fuente: Elaboración propia. 
